\title{
RAIRO-RO
}

\section{Notre ami Robert Faure}

Revue française d'automatique, d'informatique et de recherche opérationnelle. Recherche opérationnelle, tome 16, n 3 (1982), p. 277-280.

<http://www.numdam.org/item?id=RO_1982_16_3_277_0>

(C) AFCET, 1982, tous droits réservés.

L'accès aux archives de la revue « Revue française d'automatique, d'informatique et de recherche opérationnelle. Recherche opérationnelle » implique l'accord avec les conditions générales d'utilisation (http://www.numdam.org/ legal.php). Toute utilisation commerciale ou impression systématique est constitutive d'une infraction pénale. Toute copie ou impression de ce fichier doit contenir la présente mention de copyright.

\section{Numdam}

Article numérisé dans le cadre du programme

Numérisation de documents anciens mathématiques

http://www.numdam.org/ 


\section{NOTRE AMI ROBERT FAURE $\dagger$}

Notre cher et grand ami Robert Faure n'est plus. C'est un grand chagrin pour ceux qui, collègues, élèves, amis (et si souvent les trois à la fois) ont pu apprécier ses rares vertus de bienveillance et de générosité, alliées à des connaissances étendues et précises, et à une expression réunissant avec bonheur qualités scientifiques et littéraires. Il a auvré toute sa vie pour une Recherche Opérationnelle active, où tout devait concourir, avec l'aide de la mathématique et de l'informatique, à résoudre avec succès et réalisme des problèmes concrets. Il continuera à exister dans l'esprit de tous ceux qui l'ont connu. Son auvre et les travaux de ses élèves perpétueront sa mémoire à travers les jeunes générations.

J. A.

\section{ROBERT FAURE}

Ancien élève de l'École Normale Supérieure de l'Enseignement Technique; Ingénieur du Conservatoire National des Arts et Métiers (spécialité Mécanique).

Il commença à enseigner la Recherche Opérationnelle au C.N.A.M. dès 1959, bénévolement puis fut nommé chargé d'enseignement (1969), professeur associé (1971) et professeur titulaire de la chaire de R.O. (1977), spécialement créée pour lui.

Parallèlement dans l'industrie, il s'est illustré en tant que conseiller scientifique d'abord auprès de la Compagnie des Machines Bull pendant une dizaine d'années puis auprès de la R.A.T.P., pendant une autre décennie.

Pionnier de l'enseignement de la R.O., il a participé à son introduction à l'École des Mines de Paris (1960), à l'École Supérieure d'Électricité (1966), à l'E.N.S.E.T., à l'Institut Blaise Pascal (devenu depuis Institut de Programmation, Paris VI), au C.E.P.I.A., etc. Il effectua aussi de nombreuses missions tant en France qu'à l'étranger.

En collaboration avec M. DenisPapin et A. KaUfmann : Cours de calcul booléien appliqué $1^{\mathrm{re}}$ éd., 1963, $2^{\mathrm{e}}$ éd., 1970 (cours de mathématiques supérieures, t. V), Albin Michel, Paris.

Exercices de calcul matriciel et de calcul tensoriel, $1^{\mathrm{re}}$ éd., 1958, $4^{\mathrm{e}}$ éd., 1970, Eyrolles, Paris. 
Depuis septembre 1979, il avait regagné le Maroc - pays de sa jeunesse puis de son début de carrière - pour y lancer, depuis sa chaire à l'Université de Rabat, des enseignements de R.O.

Il a développé une pédagogie efficace de la R.O. fondée notamment sur l'emploi systématique des graphes, permettant de l'enseigner avec succès à de vastes auditoires, évitant de la déssécher à ses seuls aspects mathématiques. Le Professeur Faure est l'auteur de nombreux ouvrages en mathématiques, électronique et surtout Recherche Opérationnelle; en particulier du "Précis de R.O. " qui fait autorité. Il mettait la dernière main à son dernier ouvrage "Guide de la R.O », en collaboration avec le Professeur Alj, lorsque la mort l'a brutalement emporté (ce livre paraîtra chez Masson-E.A.P.).

Le Professeur Faure a mené de fructueuses recherches en Algèbre de Boole et programmation linéaire en nombres entiers; en particulier il est le co-auteur d'une des premières méthodes de recherche arborescente (publiée bien avant celles de l'école américaine).

Il était apprécié aussi pour l'étendue de sa culture générale; diplômé en philosophie et versé en arabe, il était également diplômé en berbère...

Il n'avait pas hésité à s'engager dans la lutte contre le fascisme, notamment au sein de la $2^{\mathrm{e}}$ D.B.

Au-delà de ses compétences et ses titres, il était connu pour ses qualités humaines; d'une grande humilité, il faisait preuve d'une gentillesse et d'une solidarité - bien au-delà du cadre du travail - envers toutes les personnes l'approchant, y compris les plus modestes.

Nous ne l'oublierons pas.

B. $\mathbf{L}$.

\section{BIBLIOGRAPHIE DE ROBERT FAURE}

Initiation à l'électronique $1^{\text {re }}$ éd. 1961, $2^{\mathrm{c}}$ éd. 1966, Dunod, Paris (épuisé en langue française ; disponible en langue espagnole : Iniciacion a la electronica, $2^{\mathrm{e}}$ éd. Marcombo, Barcelone).

Aide-mémoire de mathématiques nouvelles (tome 1), $1^{\text {re }}$ éd., 1970, Dunod, Paris (épuisé en français, mais disponible en langues espagnole, italienne, roumaine et, notamment en russe : Cobpemehha Matematuk, éd.; MIR, Moscou).

Précis de R.O. (4 édition complètement refondue d'Éléments de la R.O.), Paris, Dunod, 1979. Édition totalement refondue de « Éléments de la R.O. ", GauthierVillars, 1968.

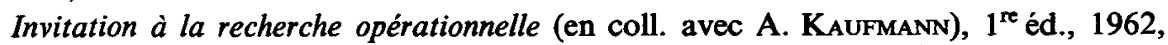
$2^{\mathrm{e}}$ éd., $5^{\mathrm{e}}$ nouveau tirage, 1976, Dunod, Paris, ouvrage traduit en 15 langues. Paris.

La programmation linéaire appliquée, Collection Que sais-je? $\mathrm{N}^{\circ} 1776,1979$, P.U.F., 
Les jeux d'entreprises (en coll. avec A. Kaufmann et A. Le GarfF), collection Que sais-je ? $\mathrm{N}^{\circ} 892,1^{\mathrm{re}}$ éd., $1960,4^{\mathrm{e}}$ éd., 1976, P.U.F. Paris.

La recherche opérationnelle (en coll. avec J. P. Boss et A. LE GARFF), $1^{\text {re }}$ éd., 1961, $5^{\mathrm{e}}$ éd., entièrement refondue (60 mille), 1980, Que sais-je? $\mathrm{N}^{\circ}$ 941, P.U.F., Paris.

En collaboration avec M. Denis-PAPIN et A. Kaufmann : Cours de calcul booléien appliqué $1^{\mathrm{re}}$ éd., 1963, $2^{\mathrm{e}}$ éd., 1970 (cours de mathématiques supérieures, t. V), Albiǹ Michel, Paris. Paris.

Exercices de calcul matriciel et de calcul tensoriel, $1^{\mathrm{re}}$ éd., 1958, $4^{\mathrm{e}}$ éd., 1970, Eyrolles,

Exercices de calcul opérationnel, $1^{\text {re }}$ éd., 1959, $3^{\mathrm{e}}$ éd., 1966 (épuisé).

Structures ordonnées et algèbres de Boole (en coll. avec E. HEURGoN), 1971, Gauthier-Villars, Paris.

Mathématiques pour l'informaticien (en coll. avec B. LemaIre), $1^{\mathrm{re}}$ éd., 1973, Gauthier-Villars, Paris.

Collection PROGRAMMATION, série RECHERCHE OPÉRATIONNELLE APPLIQUÉE.

1. Chemins et flots, ordonnancements (en coll. avec C. Roucairol et P. Tolla), 1976, Gauthier-Villars, Paris.

2. Processus stochastiques, leurs graphes, leurs usages (en coll. avec P. ChretienNe), 1974, Gauthier-Villars, Paris.

3. Éléments de programmation dynamique (J. L. LAURIERE), 1979, Gauthier-Villars, Paris.

4. Fiabilité et renouvellement des équipements (en coll. avec J. L. LAURIERE), 1974, Gauthier-Villars, Paris.

En outre, Robert Faure a publié de nombreux cours polycopiés et d'importants articles.

\section{COURS POLYCOPIÉS}

Méthodes de simulation. Informations scientifiques BULL, Paris, 1962.

Concepts essentiels de mathématique nouvelle (2 fascicules) 1963-1964, Ecole de la Compagnie des Machines BULL, modifié en 1968 à l'intention des cadres de la R.A.T.P.

Algèbre de Boole Appliquée, Institut Blaise Pascal, 1967.

Recherche Opérationnelle (cours professé à la section " Informatique ") réf. 2067 : $1^{\text {re }}$ éd., 1967, 2e éd., 1970, École Supérieure d'Électricité, Plateau du Moulon, Gif-sur-Yvette.

Compléments de mathématiques (cours professé à la section "Informatique " de l'École Supérieure d'Électricité, réf. 2536, v. ci-dessus).

Programmation linéaire en nombres entiers, Institut de Programmation, 1968.

Graphes et Applications C.N.A.M. et Institut de Programmation, 1970.

Processus stochastiques C.N.A.M. et Institut de Programmation, 1970.

Introduction à la Recherche Opérationnelle, École Supérieure d'Électricité, session de perfectionnement, 1970.

Usure Fiabilite, Institut de Programmation, 1970.

Programmation dynamique, Institut de Programmation, 1971.

Pour apprendre les fondements des mathématiques nouvelles, C.E.P.I.A., 1973.

Programmation mathématique, C.N.A.M. et l'Institut de Programmation, 1974. 
Pensez à la Recherche Opérationnelle (introduction attrayante à la R.O. pour les cadres de la R.A.T.P.), R.A.T.P., 1979.

\section{QUELQUES ARTICLES IMPORTANTS}

Programmes linéaires en nombres entiers: Méthode Booléienne; Compagnie des Machines BULL, note interne du 25-6-1962.

Une Méthode Booléienne pour la résolution des programmes linéaires en nombres entiers, Gestion, 6, 4, $\mathrm{N}^{\circ}$ spécial de R.O., p. 250-260, avril 1963.

Quelques aspects de la programmation linéaire appliquée, Colloque de calcul numérique, Lille, (6-11 juillet 1964) PST $\mathrm{N}^{\circ} 157$ du Ministère de l'Air.

Nouvelles recherches sur la résolution des programmes linéaires en nombres entiers, Gestion, 8, 6, $\mathrm{N}^{\circ}$ spécial, juin 1965.

Pour un enseignement cohérent et utile de la recherche opérationnelle, I.F.I.P., 2nd world conference; in Computers in Education, Informatique et enseignement, Lecarme et Lewis éd., 1975.

Heuristique efficace : la notion de regret en recherche opérationnelle, $3^{\mathrm{e}}$ symposium " Méthodes heuristiques », Pologne, 1976. 